\title{
Use of intrauterine balloon tamponade in successful conservative management of life threatening menorrhagia in a patient with idiopathic thrombocytopenic purpura and severe anemia
}

\section{Rekha Agrawal $^{1}$, Hemant Shintre ${ }^{1 *}$, Bindu Rani ${ }^{1}$, Krishna Agrawal ${ }^{1}$, Aniruddh Agrawal ${ }^{2}$}

\author{
${ }^{1}$ Department of Obstetrics and Gynaecology, Lilavati Hospital and Research Centre, Bandra (W), Mumbai, \\ Maharashtra, India \\ ${ }^{2}$ Topiwala National Medical College, Dr. A. L. Nair Road, Mumbai Central, Mumbai 400008, Maharashtra, India
}

Received: 02 December 2016

Accepted: 26 December 2016

\author{
*Correspondence: \\ Dr. Hemant Shintre, \\ E-mail: hemantshintre@gmail.com
}

Copyright: () the author(s), publisher and licensee Medip Academy. This is an open-access article distributed under the terms of the Creative Commons Attribution Non-Commercial License, which permits unrestricted non-commercial use, distribution, and reproduction in any medium, provided the original work is properly cited.

\begin{abstract}
A 45-year-old woman was brought into the emergency ward of a tertiary care hospital. The patient gave a history of excess bleeding and passage of clots per vaginum since the past 15 days. The patient had a history of severe headaches, body ache, giddiness and vomiting. She was diagnosed with idiopathic thrombocytopenic purpura (ITP) and severe anemia six months before this incidence. The authors performed a diagnostic pelvic and transvaginal ultrasound and reported the presence of uterine fibroids. They determined that these fibroids had aggravated the bleeding which was caused by ITP. The diagnosis of ITP coupled with severe anemia prevented the authors from using the traditional approach of a hysterectomy in such a case. She was administered tranexamic acid, testosterone enanthate, blood, intravenous immunoglobulins, steroids and platelets transfusion. Uterine artery embolization (UAE) was tried for arresting menorrhagia, but was unsuccessful. Finally, a Bakri ${ }^{\circledR}$ balloon, normally used for management of post-partum hemorrhage (PPH), was inserted and retained for 48 hrs.. The bleeding gradually ceased and significant improvements in hematocrit and platelet count were observed. However, the patient then suffered from a deep vein thrombosis (DVT) in the lower extremity, which was treated with low molecular weight heparin (LMWH), a thrombectomy and an Inferior vena cava Filter. Patient was advised a hysterectomy to curb her symptoms, however, she was too exhausted of surgical procedures and refused. Therefore, the authors decided to employ the use of Selective Estrogen Receptor Molecule Therapy (SERM). 3-months post-intervention, patient is thriving and stable.
\end{abstract}

Keywords: Bakri balloon, Deep venous thrombosis, Fibroid, Immune thrombocytopenia, Menorrhagia, Uterine artery embolization

\section{INTRODUCTION}

Chronic and extensive bleeding is a common gynecological problem in the reproductive age group. Bleeding disorders occur in a significant proportion of patients presenting with menorrhagia. Primary hemostatic dysfunction may result in abnormal uterine bleeding as a consequence of many disorders: von Willebrand's disease, qualitative platelet abnormalities, and thrombocytopenia. Studies have suggested that up to $13 \%$ of women with menorrhagia have von Wille-brand's disease and $66 \%$ of women have idiopathic thrombocytopenic purpura (ITP). ${ }^{1,2}$

A survey of gynecologists found that only $4 \%$ of practicing gynecologists would consider bleeding disorders in the differential diagnosis of menorrhagia. ${ }^{3} \mathrm{~A}$ structured history should inquire about excessive menstrual bleeding since menarche, easy bruising, and bleeding from mucous membranes could present the 
clinician with additional information. Physical examination should include a complete pelvic examination and pelvic ultrasound examination to rule out other sources of bleeding, such as vaginal lesions or uterine fibroids. Saline hysteron-sonography may also be warranted to rule out endometrial polyps if the cause remains uncertain. Recommended laboratory evaluation includes complete blood count with platelets, PT/ aPTT, pregnancy test, and thyroid stimulating hormone. A platelet function assay (e.g. ristocetin cofactor assay) should be considered if a cause is not found on initial evaluation, especially if surgical management is entertained.

In the setting where other causes of isolated thrombocytopenia have been excluded, the diagnosis of ITP should be entertained. Bone marrow aspirate and biopsy is not routinely performed in ITP except in cases of diagnostic uncertainty or in older patients to exclude diseases such as myelodysplasia. Anti-platelet antibody assays to date remain unreliable and are not routinely used. Once thrombocytopenia is established, the underlying cause must be sought.

We report a case report involving the use of Intrauterine Balloon Tamponade in Successful Conservative Management of Life Threatening Menorrhagia in a Patient with Idiopathic Thrombocytopenic Purpura and Severe Anemia.

\section{CASE REPORT}

A 45-year-old multiparous female was brought to the emergency ward of a tertiary care hospital with complaints of heavy bleeding and passage of clots per vaginum since the past 15 days. She also complained of chest pain, palpitation, giddiness, vomiting and headache which had worsened since morning. Her previous periods were regular and she said that she had observed only moderate bleeding. In the past, she had undergone three uneventful vaginal deliveries. She had also undergone a surgery for an appendectomy 20years ago, which she reported to be uneventful.

6 months before this incident, the patient was admitted to another tertiary care hospital due to complaints of weakness, body ache and giddiness. Further investigation at that time identified her as a case of idiopathic thrombocytopenic purpura (ITP) and severe anemia. Medical history reports showed that her bone marrow biopsy report was suggestive of megakaryocytic thrombocytopenia. As treatment, she was given two units of packed cell transfusion as well as steroids. Following this therapy, she had suffered from right-sided hemiplegia; however, she recovered from this condition fully.

After she was admitted to the emergency ward, on examination her general condition seemed poor. She had marked pallor with presence of cold extremities. General clinical parameters were as follows: Blood pressure $100 / 60 \mathrm{mmHg}$, heart rate was $128 / \mathrm{min}$, and the respiratory rate $24 / \mathrm{min}$. On systemic examination, tachycardia and tachypnea were observed in the cardiovascular and respiratory system, with the rest of the findings being unremarkable. On palpation, abdomen was soft and not tender. On per speculum examination, the physical appearance of the cervix seemed normal; however, blood clots were seen through cervical os. On per vaginal examination, uterus seemed bulky, irregular with bilateral fornices free.

In view of a poor general condition, she was shifted to the intensive care unit (ICU) for further management. On first day of admission, patient's hemoglobin results reported levels of $1.5 \mathrm{gm} / \mathrm{dl}$. Platelet count was reduced to $78,000 / \mathrm{mm}^{3}$. Therefore, she was immediately transfused with 4 units of packed cells. Coagulation studies revealed normal Prothrombin Time (PT), activated partial thromboplastin time (aPTT) and International Normalized Ratio (INR). To stop the excess bleeding, injection testosterone enanthate $25 \mathrm{mg}$ was administered along with tranexamic acid. However, these measures failed and the patient continued to bleed.

Diagnostic pelvic sonography was suggestive of multiple small uterine intramural fibroid (largest measuring $2.5 \mathrm{~cm}$ $\mathrm{x} 2.1 \mathrm{~cm}$ ) with endometrial thickness of $9 \mathrm{~mm}$. As the patient continued bleeding severely, bilateral uterine artery embolization (UAE) was performed on day 2 but this counter-measure too failed to stop the bleeding. The situation seemed dire, the patients hemoglobin dropped from 5.9 to $4.7 \mathrm{gm} / \mathrm{dl}$. Platelet count decreased from $78000 / \mathrm{mm}^{3}$ to $9000 / \mathrm{mm}^{3}$. Platelets transfusion (1 unit of Single Donor Platelets) was performed. IV Immunoglobulins (Ig) were also given, along with steroids for ITP. Patient refused to undergo bone marrow biopsy for further investigation.

With due risk patient was taken for dilatation and curettage and Bakri Balloon catheterization on day 3. Bakri Balloon was inflated with $50 \mathrm{ml}$ normal saline. Bleeding gradually declined over the period of 48 hours and then stopped completely. Bakri balloon deflated stepwise gradually and removed completely. Curettage done and endometrial sample send for histopathological examination which revealed secretory phase endometrium.

Steroids and IVIg were continued for ITP. Patient was shifted to ward for observation. In view of low serum ferritin levels, parenteral iron was given.

On day 7, patient developed pain in the legs with calf tenderness and was investigated for deep vein thrombosis. Findings of the color Doppler showed a partial lumen occluding thrombus at right mid-distal external iliac vein and right common femoral vein extending into great saphenous vein. Presence of mild subcutaneous edema was observed in the thigh region. 
Computed Tomography (CT) pulmonary angiography was performed and was found to be unremarkable. Patient was started on low molecular weight Heparin (LMWH). In view of previous history of thromboembolic episode, thrombectomy was performed and IVC filter was put. Patient was discharged on day 15 and advised for hysterectomy. However, at the two-week follow-up, she was too exhausted of surgical procedures and refused. Therefore, the authors decided to employ the use of Selective Estrogen Receptor Molecule Therapy (SERM). 3 -months post-intervention, patient is thriving and stable.

Table 1: Complete blood picture and peripheral smear.

\begin{tabular}{|llllllllll|}
\hline $\mathrm{Hb}(\mathrm{gm} / \mathrm{dl})$ & Day 1 & Day 1 & Day 2 & Day 3 & Day 4 & Day6 & Day 7 & Day 8 & Day 12 \\
\hline $\begin{array}{l}\mathrm{TLC} \\
\left(/ \mathrm{mm}^{3}\right)\end{array}$ & 1273 & 5.9 & 4.7 & 7.7 & 7.3 & 8.6 & 8.5 & 8.8 & 9.4 \\
\hline $\begin{array}{l}\text { Platelets } \\
\left(/ \mathrm{mm}^{3}\right)\end{array}$ & 1091 & 1882 & 1341 & 1419 & 1219 & 9990 & 1455 & 7160 \\
\hline $\begin{array}{l}\text { Peripheral } \\
\text { smear }\end{array}$ & $\begin{array}{l}\text { Microcytic } \\
\text { hypochromic, } \\
\text { anisocytosis. Pencil } \\
\text { cells, tear drop cells, } \\
\text { schistocytes }\end{array}$ & -do- & -do- & -do- & -do- & -do- & -do- & & \\
\hline
\end{tabular}

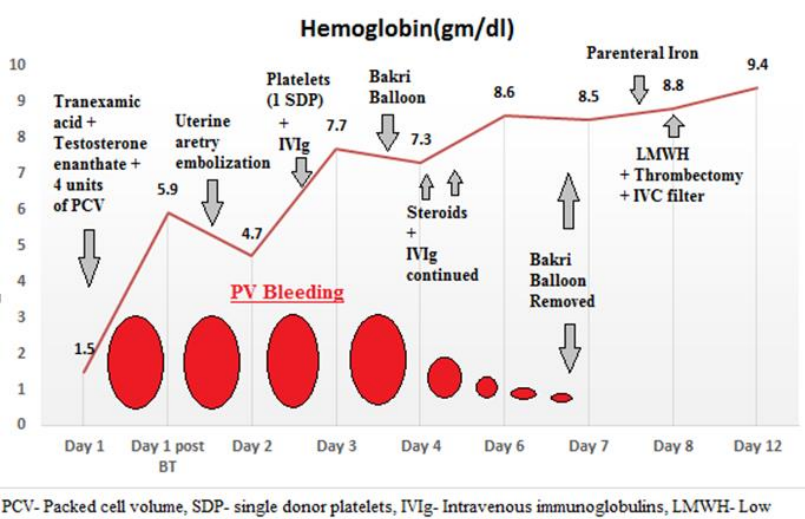

molecular weight heparin, IVC filter- Inferior vena caval filter, BT- blood transfusion, PV - per vaginal

Figure 1: Hemoglobin and PV bleeding.

\section{DISCUSSION}

Relatively few studies have evaluated the incidence of severe menorrhagia in women with thrombocytopenia. Thrombocytopenia has several etiologies, including primary bone marrow failure (aplastic anemia), bone marrow failure from myelo-suppressive chemotherapy, immune-mediated diseases (e.g. ITP) or non- immunemediated mechanisms (e.g. hemolytic uremic syndrome). ${ }^{4}$ Multiple etiologies of thrombocytopenia and a lack of objective criteria defining menorrhagia complicate any estimate of the frequency of this condition. ${ }^{5}$ One such study evaluated 71 girls aged $10-$ 19 years who presented with menorrhagia. ${ }^{2}$ The authors found that only $9(13 \%)$ had platelet counts less than $150,000 / \mathrm{L}$. Two thirds of the thrombocytopenic patients had ITP, while $22 \%$ had myelo-suppression as a result of chemotherapy, and $11 \%$ had aplastic anemia. Another study evaluated 101 premenopausal women undergoing myelo-suppressive chemotherapy who developed severe thrombocytopenia (platelet counts less than 25,000/ L). ${ }^{6}$ This study group was on average older (mean age 29.8 8.8 years) than the previous study. The sample included 20 untreated patients who had a $40 \%$ incidence of lifethreatening bleeding requiring intervention.

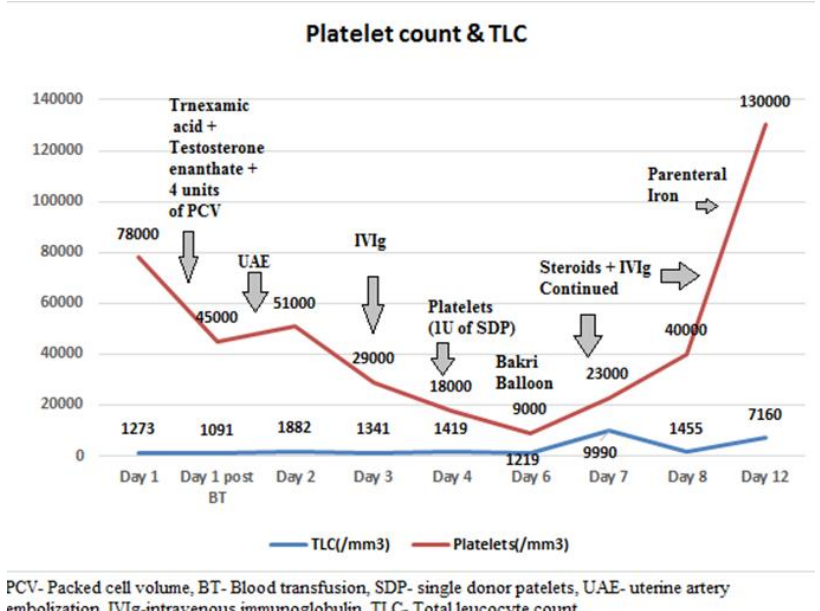

Figure 2: Platelet count and total leucocyte count.

In this report, we noted that thrombocytopenia can present transiently with severe iron deficiency anemia; however, this observation has rarely been reported. ${ }^{7-9}$ It is also noted that multimodality management involving blood transfusion, platelet transfusion, iron therapy, Bakri balloon tamponade with steroids and IVIg is the cornerstone treatment in patients who present with menorrhagia, severe iron deficiency anemia and immune thrombocytopenia. 
The exact mechanism of thrombocytopenia occurring with severe anemia in menorrhagia patients is not fully understood. Iron deficiency anemia is one of the common anaemia's and is usually associated with reactive thrombocytosis. ${ }^{10}$ Thrombocytosis, when presented with iron deficiency anemia, is suggested to be due to the stimulation of platelet production. Platelet production is induced by erythropoietin, whose levels are typically increased in patients with iron-deficiency anemia. Augmented levels of iron deficiency leads to normalization and an eventual decline in platelet numbers. The decline in platelets may be related to the variation in the activity of iron-dependent enzymes in megakaryocytosis and thrombosis. ${ }^{11}$ The pathogenesis of ITP is presumed to be related to increased platelet destruction along with inhibition of platelet production via the production of specific autoantibodies. ${ }^{12}$ Moreover, it is known that iron has both synthetic and regulatory roles in thrombopoeisis. Two additional mechanisms for low platelet count include dilutional or distributional thrombocytopenia as well as spurious or pseudo thrombocytopenia. Also, it may be noted that low platelet count may arise in immune thrombocytopenia disorders.

Uterine leiomyomas and adenomyosis are benign tumors that are mostly asymptomatic, yet can cause significant problems such as heavy uterine bleeding. This was evident in our case and was confirmed by pelvic and transvaginal ultrasound. Furthermore, atypically presented symptoms relate to the number, size, and location of the fibroid.

There is an unclear bleeding etiology with possibilities including both microscopic and macroscopic abnormalities of the uterine vasculature, impaired endometrial hemostasis, or molecular dysregulation of angiogenic factors. ${ }^{13}$ According to the PALM-COEIN (polyp; adenomyosis; leiomyoma; malignancy and hyperplasia; coagulopathy; ovulatory dysfunction; endometrial; iatrogenic and not yet classified) classification for heavy menstrual bleeding, our case can be attributed to Abnormal Uterine Bleeding (AUB)- LC (leiomyoma, coagulopathy). ${ }^{14}$

Tranexamic acid which is anti-fibrinolytic of choice was used for treatment of heavy menstrual bleeding. ${ }^{15}$ Uterine artery embolization (UAE) is a conservative approach for management of fibroids. It is good alternative for those cases who are unfit for surgery due to severe anemia and thrombocytopenia, as was ours.(16) However in our case, the UAE did not help much and patient showed steady trend of decreasing hemoglobin concentration due to ongoing blood loss. The traditional method intrauterine tamponade by balloon is commonly used for the prevention of post-partum hemorrhage. The placement of intrauterine foley catheter for 1 to 48 hours has been described as a method to temporize bleeding until hormonal/ medical therapies can take effect.(16-18) In our case, Bakri balloon tamponade played a major role in control of hemorrhage.
There is no well-defined "standard of care" for treatment of ITP in adults. The goal of therapy is to achieve hemostatic platelet count, generally considered to be 20$30 \times 10^{9} / \mathrm{L}$. Treatment is rarely required above platelet count $50 \times 10^{9} \mathrm{~L}$, and must be individualized to account for a patient's age, lifestyle, individual bleeding risk and his/her preference. Single donor platelets (SDP) are considered better than random donor platelets (RDP) as they have less chances of infection, alloimmunization and the rate of platelet survival is increased with SDP. Corticosteroids and IVIg are the first line therapy for ITP. ${ }^{19}$ In our case, platelet transfusion was given in view of active bleeding. Also the ITP responded well to steroids and IV Immunoglobulins. The platelet count started increasing and the bleeding also reduced. The Bakri balloon arrested bleeding to which allowed the clinicians to buy time for medical therapy to work.

As our patient had a history of thromboembolic episodes and was in lying down position for prolonged periods of time, she had developed deep vein thrombosis. With all these risk factor taken into account, commencement of LMWH therapy, performance of thrombectomy and placement of IVC filter was done immediately. On discharge patient was counselled for hysterectomy in view of multiple uterine fibroids.

Patient has given consent for publishing photographs, clinical history and management of the same and was assured that anonymity will be preserved.

\section{CONCLUSION}

There is no well-defined "standard of care" for treatment of ITP in adults. The goal of therapy is to achieve haemostatic platelet count. Patient should be suggested therapies while taking into account her personal situation as in case of ITP, the treatment plan should be devised individually for each patient.

Funding: No funding sources

Conflict of interest: None declared

Ethical approval: Not required

\section{REFERENCES}

1. Kadir RA, Economides DL, Sabin CA, Owens D, Lee CA. Frequency of inherited bleeding disorders in women with menorrhagia. Lancet. 1998;351(9101):485-9.

2. Bevan JA, Maloney KW, Hillery CA, Gill JC, Montgomery RR, Scott JP. Bleeding disorders: A common cause of menorrhagia in adolescents. J Pediatr. 2001;138(6):856-61.

3. Dilley A, Drews C, Lally C, Austin H, Barnhart E, Evatt B. A survey of gynecologists concerning menorrhagia: perceptions of bleeding disorders as a possible cause. J Womens Health Gend Based Med. 2002;11(1):39-44. 
4. Ewenstein BM. The pathophysiology of bleeding disorders presenting as abnormal uterine bleeding. Am J Obstet Gynecol. 1996;175(3 Pt 2):770-7.

5. Fraser IS, Bonnar J, Peyvandi F. Requirements for research investigations to clarify the relationships and management of menstrual abnormalities in women with hemostatic disorders. Fertil Steril. 2005;84(5):1360-5.

6. Meirow D, Rabinovici J, Katz D, Or R, Shufaro Y, Ben-Yehuda D. Prevention of severe menorrhagia in oncology patients with treatment-induced thrombocytopenia by luteinizing hormone-releasing hormone agonist and depo-medroxyprogesterone acetate. Cancer. 2006;107(7):1634-41.

7. Ibrahim R, Alhilli JA, Cooper TT, Dashkova I, Guy J, Gandhi A, et al. Idiopathic thrombocytopenia with iron deficiency anemia. Clinical medicine insights Blood disorders. 2013;6:1-5.

8. Morris VK, Spraker HL, Howard SC, Ware RE, Reiss UM. Severe thrombocytopenia with iron deficiency anemia. Pediatric hematology and oncology. 2010;27(5):413-9.

9. Ibrahim R, Khan A, Raza S, Kafeel M, Dabas R, Haynes E, et al. Triad of iron deficiency anemia, severe thrombocytopenia and menorrhagia-a case report and literature review. Clinical medicine insights Case reports. 2012;5:23-7.

10. Patel KV, Harris TB, Faulhaber M, Angleman SB, Connelly S, Bauer DC, et al. Racial variation in the relationship of anemia with mortality and mobility disability among older adults. Blood. 2007;109(11):4663-70.

11. Schafer AI. Thrombocytosis. The New England journal of medicine. 2004;350(12):1211-9.

12. Provan D, Stasi R, Newland AC, Blanchette VS, Bolton-Maggs $\mathrm{P}$, Bussel JB, et al. International consensus report on the investigation and management of primary immune thrombocytopenia. Blood. 2010;115(2):168-86.

13. Parker WH, Iacampo K, Long T. Uterine rupture after laparoscopic removal of a pedunculated myoma. Journal of minimally invasive gynecology. 2007;14(3):362-4.

14. Munro MG, Critchley HO, Broder MS, Fraser IS, Disorders FWGoM. FIGO classification system (PALM-COEIN) for causes of abnormal uterine bleeding in nongravid women of reproductive age. International journal of gynaecology and obstetrics: the official organ of the International Federation of Gynaecology and Obstetrics. 2011;113(1):3-13.

15. Naoulou B, Tsai MC. Efficacy of tranexamic acid in the treatment of idiopathic and non-functional heavy menstrual bleeding: a systematic review. Acta obstetricia et gynecologica Scandinavica. 2012;91(5):529-37.

16. Vilos GA, Allaire C, Laberge PY, Leyland N, Special C, Vilos AG, et al. The management of uterine leiomyomas. Journal of obstetrics and gynaecology Canada : JOGC = Journal d'obstetrique et gynecologie du Canada : JOGC. 2015;37(2):15781.

17. Goldrath MH. Uterine tamponade for the control of acute uterine bleeding. American journal of obstetrics and gynecology. 1983;147(8):869-72.

18. Hamani Y, Ben-Shachar I, Kalish Y, Porat S. Intrauterine balloon tamponade as a treatment for immune thrombocytopenic purpura-induced severe uterine bleeding. Fertility and sterility. 2010;94(7):2769 e 13-5.

19. Kistangari G, McCrae KR. Immune thrombocytopenia. Hematology/oncology clinics of North America. 2013;27(3):495-520.

Cite this article as: Agrawal R, Shintre H, Rani B, Agrawal K, Agrawal A. Use of intrauterine balloon tamponade in successful conservative management of life threatening menorrhagia in a patient with idiopathic thrombocytopenic purpura and severe anemia. Int J Reprod Contracept Obstet Gynecol 2017;6:761-5. 